\title{
REA_09 - Development of monoclonal antibodies targeting SARS-CoV-2 Spike and Nucleocapsid proteins
}

Milena Mouta Verdan França Carvalho ${ }^{1 *}$; Thiago dos Santos Chaves ${ }^{1}$; Rodrigo Nunes Rodrigues da Silva ${ }^{1}$; Bruno Vinícius da Conceição Souza ${ }^{1}$; Rodrigo Muller ${ }^{1}$; Gabriela dos Santos Esteves ${ }^{1}$; Renata Chagas Bastos $^{1}$; Adriana de Souza Azevedo Soares ${ }^{1}$; Sotiris Missailidis ${ }^{1}$; Patrícia Cristina da Costa Neves ${ }^{1}$. ${ }^{1}$ Fiocruz/Bio-Manguinhos;

Introduction: In December 2019, SARS-CoV-2 virus jumped the barrier between species and started to infect humans in Wuhan province, China. The disease has spread quickly and has caused thousands of deaths since the declaration of the Covid-19 pandemic by the WHO. In order to avoid the spread of the disease, mass diagnosis is essential as a strategy for public health, allowing immediate quarantine of infected people. Added to this, the discovery of an effective treatment brings hope to those currently infected. Herein we have developed antibodies against Spike and Nucleocapsid proteins of SARS-CoV-2 which could help the development of antigen capture point-of-care tests and treatment of this illness.

Objective: Development of monoclonal antibodies (mAbs) targeting SARS-CoV-2 Spike and Nucleocapsid proteins.

Methodology: Balb/c mice (CEUA LW-13/16) were immunized using recombinant Spike (S) and Nucleocapsid (N) proteins (two groups). After immunization, spleens were collected and splenocytes were fusioned to myeloma SP2/0 cells, generating hybridomas, according to hybridoma standard protocols. After the selection and cloning steps, clones were expanded, cryopreserved (seed banks) and the antibodies produced in high yielding performance flask (Hyperflask) cell culture vessels. The supernatants were purified and subjected to biochemical characterization: isotyping, titration, plaquereduction neutralization tests (PRNT) and sequencing.

Results: After scanning more than 300 clones, we have selected 6 clones against S protein and 5 clones against $\mathrm{N}$ protein. Isotyping tests revealed that clones are IgG1 (4), IgG3 (1), IgG1/2a (1), IgM (4) and IgA (1). For S protein, ELISA tests have shown that 3 of clones are specific to 2 distinct regions of S1, 2 for RBD and 1 for S2 region. In addition, to spike protein, clones have shown titers ranging 1:1.600 1:200.000 and 1:12.000 - 1:25.000 to RBD. In addition, viral neutralization assay showed that 2 clones are neutralizing, based on a $50 \%$ reduction in plaque counts $\left(\mathrm{PRNT}^{50}\right)$.

Conclusion: Taken together, these results demonstrated that we have successfully developed at least 11 different clones against SARS-CoV-2 proteins that may be important tools for antigen capture diagnosis tests development and treatment of the disease.

Keywords: SARS-CoV-2; monoclonal antibodies; diagnostic 